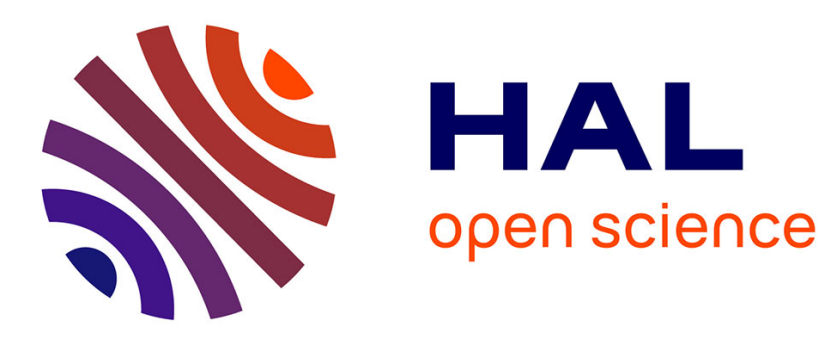

\title{
Loi du marché et discrimination
}

Danièle Lochak

\section{To cite this version:}

Danièle Lochak. Loi du marché et discrimination. Daniel Borrillo. Lutter contre les discriminations, La Découverte, pp. 11-37, 2003, 9782707140272. hal-01698163

\section{HAL Id: hal-01698163 https://hal.parisnanterre.fr/hal-01698163}

Submitted on 31 Jan 2018

HAL is a multi-disciplinary open access archive for the deposit and dissemination of scientific research documents, whether they are published or not. The documents may come from teaching and research institutions in France or abroad, or from public or private research centers.
L'archive ouverte pluridisciplinaire HAL, est destinée au dépôt et à la diffusion de documents scientifiques de niveau recherche, publiés ou non, émanant des établissements d'enseignement et de recherche français ou étrangers, des laboratoires publics ou privés. 


\title{
Loi du marché et discrimination
}

\author{
par Danièle Lochak
}

in D. Borrillo (dir.), Lutter contre les discriminations, La découverte, 2003, pp. 11-37.

Non seulement le marché favorise les discriminations, mais la discrimination est inhérente au fonctionnement même du marché. Tel est le constat - non pas pessimiste, mais réaliste - auquel conduit une observation lucide des choses, et qui explique les obstacles auxquels se heurte toute tentative d'éradiquer les discriminations dans la sphère marchande.

À première vue, l'affirmation peut paraître paradoxale : marché et discrimination ne sont-ils pas antinomiques ? le marché n'implique-t-il pas la libre concurrence, laquelle à son tour dénote et connote une situation dans laquelle les concurrents s'affrontent à armes égales ? Le marché, affirme Hayek, repose sur l'égalité formelle, puisque les règles qu'il comporte sont des règles impersonnelles, abstraites, objectives, identiques pour tous.

On sait, bien sûr, que l'égalité formelle a peu de choses à voir avec l'égalité réelle dont elle n'est une condition ni suffisante, ni nécessaire ; on sait aussi que la liberté sur laquelle est basée l'économie de marché non seulement laisse subsister les inégalités sociales, mais les renforce - et Hayek lui-même convient que, dans la société de concurrence, «le pauvre a beaucoup moins de possibilités que le riche ». Ce constat banal est difficilement récusable.

Aussi bien notre propos n'est-il pas de démontrer l'incapacité du marché à assurer l'égalité des conditions, mais de montrer qu'il n'assure même pas l'égalité de traitement, en mettant en lumière les phénomènes de discrimination qu'il induit. Il s'agit de pointer non pas les conséquences d'un effet de système : les inégalités engendrées ou entretenues par le système capitaliste, mais les comportements conscients d'agents économiques déterminés qui réservent des traitements différenciés à des individus catégorisés sur la base de critères dictés par une logique de profit. L'employeur, l'assureur, le banquier, le logeur: tous sont amenés, dans l'exercice de leur activité, à choisir, sélectionner, écarter les candidats qui viennent solliciter un emploi, une assurance, un crédit, un logement ; pour opérer ce tri, ils sont évidemment enclins, en l'absence de toute contrainte légale, à éliminer, conformément à l'intérêt de l'entreprise, ceux qui risquent de leur faire perdre de l'argent ou de les affaiblir face à la concurrence, c'est-à-dire, en pratique, les individus appartenant aux catégories les plus vulnérables.

Non seulement les principes du libéralisme économique qui régissent le fonctionnement de la sphère marchande laissent la porte ouverte aux discriminations (1), mais la recherche du profit sous la contrainte de la concurrence y pousse inéluctablement (2). L'intervention de l'État, nécessaire pour échapper à la « loi d'airain »du marché lorsqu'elle entrave l'exercice de droits fondamentaux, ne peut se borner à mettre en place un arsenal répressif contre les discriminations (3). 


\section{1/ Le libéralisme économique fonde la liberté de discriminer}

Le libéralisme réclame, pour les acteurs économiques, la liberté la plus large : la liberté de jouir de sa propriété et de disposer librement de son bien, la liberté d'acheter ou de vendre, d'échanger des biens et des services, la liberté de contracter, qui donne corps aux précédentes. Ce faisant, il laisse le champ libre aux discriminations.

Car si la liberté est proclamée au bénéfice de tous, tous ne sont pas en mesure d'en profiter au même degré. La liberté d'entreprendre, par exemple, implique le libre choix des salariés par l'employeur; face à elle, la liberté du travail, qui implique le droit de choisir librement son activité, et donc, pour le candidat à l'embauche, le droit de ne pas accepter l'emploi qu'on lui propose, ne fait guère le poids. Quant à la liberté contractuelle, bien que le contrat soit fondé sur le principe de l'égalité des contractants, elle n'est souvent pleine et entière que pour l'une des parties. C'est cette dissymétrie qui ouvre la voie aux pratiques de discrimination.

\section{La liberté contractuelle : une liberté dissymétrique...}

La liberté contractuelle est la concrétisation la plus aboutie de l'autonomie de la volonté et l'expression par excellence de la philosophie individualiste : l'individu est obligé uniquement parce qu'il l'a voulu et dans la mesure où il l'a voulu. Les théoriciens du libéralisme économique, de leur côté, voient dans le libre jeu des volontés individuelles le moteur de la régulation par le marché et le moyen d'un ajustement harmonieux entre les intérêts des agents économiques.

La liberté contractuelle joue à trois niveaux : elle implique la liberté de s'engager ou non dans les liens du contrat, le libre choix du cocontractant, la libre détermination du contenu du contrat. La liberté contractuelle n'a certes jamais été absolue puisque, déjà, les rédacteurs du code civil avaient subordonné la validité du contrat à l'existence d'un objet et d'une cause licites. Au fil du temps, elle a subi des restrictions croissantes qui l'ont affectée dans l'ensemble de ses dimensions : le législateur est intervenu pour réglementer le contenu des contrats, imposer certaines clauses, en interdire d'autres ; il a supprimé dans certains cas la faculté de ne pas contracter (obligation d'assurance), ou encore la liberté de choisir son cocontractant (droit de préemption).

Mais même ainsi limitée et encadrée, la liberté contractuelle n'en continue pas moins à présider au fonctionnement de la sphère marchande ; or cette sphère marchande est caractérisée par l'inégalité structurelle entre les parties qui vont être amenées à contracter. Le législateur n'est pas resté aveugle à ce constat : le droit du travail, le droit de la consommation, dans une moindre mesure le droit des assurances ont précisément pour objectif de protéger la partie la plus faible contre la partie la plus forte. Mais il n'est pas dans le pouvoir du législateur d'abolir cette inégalité, ni même d'en supprimer les effets, qu'il peut seulement atténuer.

En apparence, la liberté contractuelle s'applique aux deux parties : si l'employeur, l'assureur, le banquier, le logeur ont le choix de contracter ou non avec tel ou tel, de son côté, le salarié n'est pas obligé de s'embaucher, le client potentiel n'est pas obligé de s'assurer ou de contracter un emprunt aux conditions qu'on lui propose, le locataire en puissance n'est pas obligé de louer le logement qu'on lui offre. Mais on sait bien que cette symétrie n'est que théorique, que la liberté de contracter n'est souvent, pour la partie la plus faible, qu'une liberté purement formelle, une 
liberté sous contrainte, une liberté factice. Car le besoin de contracter n'a pas la même intensité, n'est pas de même nature, de part et d'autre. Pour le candidat à l'emploi, à un logement, à l'assurance, à un crédit, le besoin de contracter revêt souvent un caractère vital. Juridiquement, certes, on peut choisir de ne pas travailler, de ne pas s'assurer, de ne pas louer un logement, de ne pas contracter un crédit. Mais dans la pratique il y a bien une contrainte, qui n'existe pas pour l'autre partie: car, sauf cas particulier (l'assurance automobile), il n'y a pas de droit à l'assurance, opposable à l'assureur, pas de droit au crédit opposable au banquier ; quant au droit à l'emploi ou au logement, bien que proclamés comme principes généraux, ils ne se concrétisent pas dans un droit à obtenir l'emploi ou le logement postulé.

L'employeur peut donc user pleinement de sa liberté contractuelle : ses prérogatives de chef d'entreprise lui confèrent toute latitude pour décider de recruter tel candidat plutôt que tel autre. De même, l'assureur est libre de fixer les conditions auxquels il accepte d'assurer un client d'autant plus libre que le contrat d'assurance est un contrat d'adhésion. Les compagnies ne sont jamais tenues d'assurer un candidat à l'assurance, sauf abus caractérisé tombant sous le coup du refus de vente ou de prestation de service. Elles ont le droit, en particulier, de refuser un candidat « trop risqué » (voir plus loin), ou de le prendre en compte sous forme de « risque aggravé », impliquant une supprime plus ou moins élevée.

Il y a bien sûr des hypothèses où la concurrence joue son rôle de régulation : à l'instar du consommateur qui, face à des commerçants dispersés, finit par trouver le produit offrant le meilleur rapport qualité-prix, le client pourra trouver auprès d'une autre compagnie ou d'une autre banque l'assurance ou le crédit que la première lui a refusé, le salarié pourra choisir parmi les offres d'emploi celle qui lui procure les conditions de travail et de rémunération les plus intéressantes. Encore faut-il qu'il y ait effectivement place pour ce jeu de l'offre et de la demande. Or l'individu à la recherche d'un emploi, d'une assurance, d'un crédit, ou même d'un logement est rarement à même de mettre en concurrence les entreprises ou les personnes auxquelles il s'adresse. C'est lui, au contraire, qui, la plupart du temps, va se trouver en concurrence avec d'autres, de sorte que c'est l'entreprise qui pourra faire jouer à son profit la concurrence entre les postulants. On pense évidemment aux demandeurs d'emplois en concurrence pour une même offre, ou aux candidats à un logement dans le contexte d'une pénurie de logements disponibles. Mais la concurrence existe aussi, sous d'autres formes, pour l'accès à des biens ou services a priori non contingentés, comme le crédit ou l'assurance, car les banquiers comme les assureurs sont à la recherche des clients présentant le profil le plus avantageux, qu'ils s'efforcent d'attirer en leur offrant des conditions plus favorables.

Loin de conforter l'égalité entre les contractants, la concurrence renforce au contraire le caractère dissymétrique de la liberté contractuelle et ouvre la voie aux discriminations.

\section{... qui inclut la " liberté de discriminer »}

L'expression «liberté de discriminer » peut paraître entachée d'une contradiction dans les termes. La discrimination n'est-elle pas prohibée aujourd'hui par une série de normes juridiques de plus en plus nombreuses, n'est-elle pas considérée comme ce qui est fondamentalement à

proscrire ? Comment, dans ces conditions, parler d'une « liberté » de discriminer ? Il convient donc de revenir un instant sur la notion de discrimination et ses différentes acceptions.

Au sens le plus large, peut être qualifiée de discrimination toute différence de traitement, qu'elle soit inscrite dans un texte ou qu'elle résulte du comportement de telle personne ou telle 
institution. Cette acception est conforme à l'étymologie, puisque discriminer, au sens propre, ce n'est rien d'autre qu'opérer une distinction, une séparation, une différenciation entre des objets.

Mais le mot discrimination est assez rarement employé dans son sens premier et neutre : discriminer, dans le langage courant, ce n'est pas simplement distinguer, mais simultanément hiérarchiser, traiter de façon moins favorable ceux qui, précisément, seront dits victimes d'une discrimination. Est qualifié de «discriminatoire» le comportement ou l'attitude qui tend à distinguer des autres un groupe humain ou une personne, à son détriment.

Le langage juridique a dans l'ensemble intégré cette connotation négative, comme en témoigne la volonté de plus en plus nette, tant au niveau international que dans les législations internes, de traquer et de mettre hors-la-loi toutes les formes de «discrimination ». Même si un certain flottement terminologique conduit parfois à qualifier de discrimination toute différence de traitement opérée entre des catégories de personnes, indépendamment de son caractère licite ou non, la discrimination apparaît bien comme la distinction ou la différence de traitement illégitime, celle que l'on veut proscrire'.

Mais comment décidera-t-on qu'une distinction ou une différence de traitement est illégitime ? Ici encore, deux conceptions coexistent. Dans une conception extensive, la discrimination se résume dans la violation du principe d'égalité : il $\mathrm{y}$ a discrimination, non pas dès l'instant où est instaurée une différence de traitement, puisque le principe d'égalité n'impose pas de traiter tout le monde de façon identique, mais dès lors que cette différence de traitement n'est pas justifiée par une différence de situation. C'est l'optique de la jurisprudence du Conseil constitutionnel, du Conseil d'État, ou encore de la Cour européenne des droits de l'homme (qui admettent toutefois qu'il puisse être dérogé au principe d'égalité pour un motif d'intérêt général prééminent). Dans une conception plus restrictive, le terme « discrimination » désigne le traitement défavorable dont sont victimes des personnes particulièrement vulnérables en raison de leur appartenance à un groupe défini par une caractéristique particulière (le sexe, la race ou l'origine ethnique, le handicap, l'orientation sexuelle...) : c'est l'optique du code pénal français, mais aussi du code du travail, ou encore de la réglementation communautaire depuis le Traité d'Amsterdam.

L'enjeu est loin d'être simplement conceptuel : la prohibition des discriminations a une portée beaucoup plus large dans le premier cas que dans le second, comme l'illustre la comparaison entre les règles qui régissent l'accès à la fonction publique et l'embauche dans le secteur privé. La conception rigoureuse du principe d'égalité qui prévaut dans l'administration oblige à respecter des critères de sélection objectifs concrétisés par la règle du concours, tandis que l'employeur est libre d'utiliser les critères de recrutement qu'il veut, du moment que ces critères ne sont pas considérés comme discriminatoires par le code du travail ou le code pénal.

C'est ce décalage entre deux conceptions du principe de non-discrimination qui autorise à parler de «liberté de discriminer »: on veut signifier par là que les personnes privées sont libres

- On retrouve jusque dans le code pénal ce flottement terminologique. En effet, le code pénal définit dans son article 225-1 la discrimination comme « toute distinction » opérée sur la base d'un des critères qu'il énumère ; mais l'article 225-2 ne sanctionne la discrimination que lorsqu'elle consiste dans un comportement déterminé (refus de vente, refus d'embauche, etc.). On peut en inférer qu'il y a des discriminations permises, ou à tout le moins non prohibées : ce que confirme l'article 225-3, qui fait échapper à la sanction pénale « les discriminations fondées sur l'état de santé » opérées par les assureurs et celles qui aboutissent à exclure d'un recrutement un candidat inapte au travail (sur ce point, v. infra, 3). 
d'adopter des comportements qui, s'ils étaient le fait d'une personne publique, seraient qualifiés de discriminatoires et interdits, car attentatoires au principe d'égalité.

En effet, contrairement à l'administration et aux services publics en général, les personnes privées - particuliers ou entreprises - ne sont pas tenues de respecter un principe général d'égalité, et le droit positif ne leur interdit pas de traiter avec autrui sur une base inégalitaire aussi longtemps qu'elles ne violent aucune prohibition précise résultant des textes. Le principe d'égalité cède ici devant le principe de liberté. La liberté du propriétaire, la liberté de l'employeur, la liberté du prestataire de service : autant d'expressions du principe civiliste de la liberté contractuelle qui inclut la faculté de traiter différemment, et même de façon inégale, en vertu de critères qu'ils déterminent librement, les individus avec lesquels ils s'engagent dans des relations contractuelles.

Le propriétaire peut louer son bien à qui bon lui semble, dès lors qu'il n'exclut personne en raison de sa race, de sa religion, ou d'un autre critère prohibé par le code pénal. Rien n'interdit au bailleur de louer son appartement à un fonctionnaire célibataire plutôt qu'à une famille nombreuse vivant avec un seul salaire, a fortiori à un chômeur. L'employeur, au nom du pouvoir de direction qui lui est reconnu, définit librement sa politique de sélection du personnel, aussi longtemps, là encore, et comme on l'a rappelé plus haut, qu'il ne se fonde pas sur des critères prohibés. Le prestataire de services a le libre choix de ses clients : c'est dans ce contexte que le banquier va pratiquer le «scoring », que l'assureur va se livrer à la « sélection des risques » (v . infra, 2). Le principal frein aux pratiques discriminatoires provient des règles sur la concurrence ; mais celles-ci, destinées essentiellement à encadrer les rapports horizontaux entre entreprises, ne protègent les particuliers - essentiellement les consommateurs - de façon très partielle.

Ces pratiques sélectives n'étant pas illicites, elles ne peuvent pas être qualifiées de discriminatoires, au sens juridique du terme. Mais le mécanisme de discrimination, lui, est bien réel, dès lors que la mise en œuvre de critères « discriminants » débouche sur un traitement non seulement différencié mais inégal des personnes concernées.

\section{2/ La loi du marché pousse irrésistiblement à la discrimination}

Libres de discriminer, les agents économiques sont de surcroît irrésistiblement poussés à le faire par la loi du marché. Dans un système capitaliste, les entreprises doivent rendre des comptes à leurs actionnaires: elles sont donc tenues de faire du profit. Dans un environnement concurrentiel, elles sont à la recherche de tout ce qui peut constituer un avantage et, inversement, prennent garde d'éviter tout ce qui risquerait de représenter une charge ou un handicap.

C'est sur la base de critères définis en fonction de ces exigences qu'elles vont sélectionner salariés et clients. Ce tri, dicté par la recherche du profit sous la contrainte de la concurrence, aboutit à écarter les personnes à qui manque une «qualité » ou affectées d'un « défaut », bref, marquées par une caractéristique qui les rend de façon générale plus vulnérables sur le plan social. La discrimination - on s'en doutait - vient la plupart du temps redoubler des inégalités préexistantes. 


\section{Un tri dicté par la recherche du profit sous la contrainte de la concurrence...}

Tout chef d'entreprise souhaite légitimement s'attacher les services des salariés les plus performants et écarter à l'inverse les moins productifs ou ceux qui pourraient faire fuir la clientèle. Tout chef d'entreprise, de même, lorsqu'il décide d'investir ou accepte de prendre un risque financier, se détermine en fonction des bénéfices qu'il espère en retirer à plus ou moins long terme.

Pour prendre ces décisions, l'outil informatique est de plus en plus souvent sollicité. Les profils statistiques, dans la mesure où ils permettent de prendre simultanément en considération une multitude de paramètres, occultent d'une certaine façon la discrimination, alors qu'ils n'en sont qu'une forme plus sophistiquée. La « sélection des risques » pratiquée par les assureurs, en revanche, se donne à voir ouvertement comme un mécanisme de discrimination, qu'on justifie par les principes économiques qui fondent l'assurance.

\section{Les profils statistiques, ou la discrimination occultée}

Des systèmes d'aide à la décision reposant sur la méthode des profils statistiques sont couramment utilisés dans le domaine du recrutement, ou par les banques pour l'octroi de crédits. Les logiciels d'aide à la sélection du personnel, par exemple, fonctionnent à partir de questionnaires visant à obtenir une description exhaustive du candidat, d'où se dégage un « profil », qu'on peut alors comparer au profil prédéterminé pour le poste à pourvoir ainsi qu'à celui des autres demandeurs. Les organismes de crédit, de leur côté, ont recours de façon systématique, surtout pour les prêts à la consommation, au «scoring », qui vise à évaluer les risques que comporte l'octroi d'un prêt en fonction d'une série de paramètres combinés.

Ces outils, qui permettent d'harmoniser les critères de sélection ou de tarification, doivent en principe rester une simple aide à la décision et non pas se substituer à elle. Rappelons en effet qu'aux termes de la loi «Informatique et libertés» du 6 janvier 1978, «aucune décision administrative ou privée impliquant une appréciation sur un comportement humain ne peut avoir pour seul fondement un traitement automatisé d'informations donnant une définition du profil ou de la personnalité de l'intéressé » (art. 2). En pratique, cette disposition est facile à tourner, et la plupart du temps la méthode des profils, en particulier le « scoring », s'apparente à un mécanisme de tri automatique, le résultat fourni par l'ordinateur étant à peu près systématiquement entériné par l'autorité à qui incombe la décision.

Les organismes de crédit ont depuis longtemps procédé à des études de solvabilité de la clientèle et établi le profil du «mauvais payeur». La méthode a été progressivement perfectionnée et aujourd'hui, à l'occasion d'une demande de crédit, un «score » est affecté au demandeur à partir d'une grille d'informations : l'âge, la situation familiale, la qualité par rapport au logement, la vie professionnelle, la situation économique ou financière, enfin les biens ou services faisant l'objet de la demande de prêt. Chacune de ces informations est affectée d'une note positive ou négative, qui permet d'évaluer automatiquement, à partir de calculs statistiques et de probabilités, la capacité de remboursement du candidat au crédit et les risques d'impayés. Et c'est sur la base de la combinaison de ces critères - le «scoring » - qu'est prise la décision d'octroyer ou non le prêt et qu'en sont fixées les conditions. Par égard pour la loi « informatique et libertés », qui impose qu'il y ait au moins formellement une intervention humaine au stade de la prise de décision, et aussi parce que l'organisme de crédit peut avoir lui-même intérêt à corriger les résultats trop brutaux du tri informatique et à « repêcher » un candidat éliminé, il est 
toujours possible d'accepter un demandeur dont le score aurait conduit à une décision de refus (et inversement). Cette possibilité, dite «forçage», représente toutefois, de l'aveu même des organismes concernés, moins de $1 \%$ des décisions prises dans le cadre du crédit à la consommation.

Le caractère impersonnel et automatisé de la prise de décision masque la discrimination qui sous-tend le fonctionnement de ce mécanisme de sélection. La combinaison, selon un algorithme mathématique, des critères pris en compte pour établir des «profils de risque » empêche qu'apparaissent en pleine lumière le ou les éléments qui ont provoqué l'élimination d'une candidature'. Et pourtant, ce ou ces éléments existent bien, que ce soit un salaire trop faible, un nombre d'enfants à charge trop élevé, un emploi précaire, ou encore la nationalité.

« De la sélection de la clientèle à la discrImination » : c'est en ces termes que la CNIL ellemême pointait, dans son 19` rapport d'activité pour 1998, les risques inhérents au scoring³. Elle visait, en l'espèce, la prise en compte de la nationalité comme variable discriminante du score : soit en tant que telle, lorsque certaines nationalités ou appartenances géographiques sont considérées comme «statistiquement risquées », soit sous une forme atténuée consistant à attribuer une pondération différente selon la répartition: Français, Union européenne, autres. Dans sa délibération du 22 décembre 1998 relative à la gestion des crédits ou des prêts consentis à des personnes physiques par les établissements de crédit, la CNIL a considéré que la nationalité n'était pas une donnée pertinente et qu'elle ne pouvait donc entrer en ligne de compte, même sous une forme atténuée, dans le calcul automatisé de l'appréciation du risque. Dans la mesure où, pour justifier cette catégorisation, les banques mettaient en avant les difficultés de recouvrement des impayés lorsque les personnes résident à l'étranger, elle a en revanche admis que pouvait être prise en compte la stabilité de la résidence du demandeur de crédit sur le territoire français (durée du titre de séjour, par exemple).

C'est dans ce contexte qu'a pris naissance le litige opposant les banques à la CNIL, arbitré par le Conseil d'État en faveur des premières ${ }^{4}$. Les motifs invoqués pour annuler la délibération de la CNIL sont à beaucoup d'égards contestables, et même dangereux. Car en admettant sans restriction aucune que la nationalité puisse constituer un élément d'appréciation d'éventuelles difficultés de recouvrement des créances, et donc une donnée pertinente au regard de la finalité du traitement, le Conseil d'État légitime par avance les pratiques discriminatoires des organismes de crédit, que rien ne retiendra plus, à l'avenir, d'écarter les demandes émanant des ressortissants étrangers.

Mais le raisonnement du Conseil d'État est aussi symptomatique de l'effet d'occultation produit par le recours aux profils. On pouvait en effet soutenir avec quelque vraisemblance que la prise en compte du critère de la nationalité tombait sous le coup de l'article 225-1 du code pénal, qui sanctionne le refus de fournir un bien ou un service en raison de l'appartenance ou de la nonappartenance d'une personne à une nation déterminée, et que la distinction entre Français et

2La loi «informatique et libertés » prévoit, certes, que «toute personne a le droit de connaître et de contester les informations et les raisonnements utilisés dans les traitements automatisés dont les résultats lui sont opposés » (art. 3), mais cette faculté n'est guère utilisée en pratique.

CNIL, 19 rapport d'activité 1998, La Documentation française, 1999, p. 123.

CE, Section, 30 octobre 2001, Association français des sociétés financières et autres, $\mathrm{n}^{\circ} 204909$, Les Petites Affiches, 1" novembre 2001, $\mathrm{n}^{\circ} 218$; voir aussi Le Monde, 1* novembre 2001 : «Le Conseil d'État autorise les banques à prendre en compte la nationalité des consommateurs dans l'octroi d'un crédit ». 
ressortissants communautaires violait le principe de non-discrimination qui sous-tend l'ensemble du droit communautaire. Le Conseil d'État écarte les deux objections d'un revers de main sans même juger nécessaire de les réfuter au fond. "La référence à la nationalité comme l'un des éléments de pur fait d'un calcul automatisé du risque[souligné par nous], dont la mise en œuvre n'entraîne pas le rejet d'une demande sans l'examen individuel de celle-ci, ne constitue pas une discrimination » au sens du droit communautaire, et, en l'absence d'élément intentionnel, elle ne tombe pas non plus sous le coup du code pénal.

Le Conseil d'État affirme ici plusieurs choses, en partie contradictoires : d'un côté, il se fonde sur l'existence d'un examen individuel des demandes, en aval du «scoring », pour écarter l'allégation d'une discrimination qui résulterait de la seule mise en œuvre du traitement incluant la variable «nationalité » (argument passablement hypocrite puisque, dans ses conclusions, la commissaire du gouvernement indiquait que le «forçage » concerne moins de $1 \%$ des dossiers) ; de l'autre, il met au contraire en avant le fait que la prise en compte de la nationalité est un élément «de pur fait» d'un «calcul automatisé », donc l'absence de volonté délibérée de discriminer, pour écarter l'allégation d'un comportement délictuel. Dans un cas l'automaticité est niée, dans l'autre elle est mise en exergue; et dans tous les cas est occultée cette vérité d'évidence qu'il a bien fallu, pour que le critère de la nationalité soit pris en compte dans un traitement, même automatisé, que le concepteur du traitement en décide ainsi...

\section{La « sélection des risques » dans le champ de l'assurance}

La discrimination trouve, dans le champ de l'assurance, un terreau particulièrement propice à son développement, dans la mesure où il est de l'essence même du contrat d'assurance de sélectionner les risques. La remarque s'applique essentiellement aux assurances de personnes, en particulier aux contrats d'assurance-vie, mais aussi à l'assurance maladie ou invalidités.

Le contrat d'assurance-vie est «le contrat par lequel l'assureur s'engage envers le souscripteur, moyennant une prime, à verser une somme déterminée au bénéficiaire désigné, l'exécution de son obligation dépendant de la durée de vie de l'assuré $»^{6}$. L'assureur va donc faire dépendre les conditions du contrat de la sélection des risques de décès que présente l'assuré, en prenant en considération à la fois les facteurs généraux qui influent sur la mortalité (âge, sexe, profession, climat), et les facteurs particuliers tels que son état de santé et ses antécédents personnels ou familiaux.

Comme le rappelle Yvonne Lambert-Faivre, la sélection des risques par l'assureur est parfaitement conforme à la technique de l'assurance, qui a pour fondement la recherche individuelle de sécurité par la mutualisation des risques". Les assureurs sont des «vendeurs de sécurité », et le coût de la sécurité est fonction du risque présenté par l'assuré. Il n'est pas excessif d'affirmer que la discrimination est partie intégrante du contrat d'assurance-vie (comme,

\footnotetext{
${ }^{5}$ On distingue les assurances de dommages et les assurances de personnes. Celles-ci couvrent les risques susceptibles d'affecter la personne humaine de l'assuré, soit dans son existence (assurances sur la vie), soit dans son intégrité physique (assurances dommages corporels, invalidité, accidents, maladie).

${ }^{6}$ Yvonne Lambert-Faivre, Droit des assurances, Précis Dalloz, 11 éd., 2001, p. 719.

${ }^{7}$ Op. cit. p. 733 .
} 
du reste, du contrat d'assurance invalidité ou maladie), car les principes économiques sur lesquels se fonde l'assurance de personnes poussent inéluctablement à la discrimination .

L'assurance repose en premier lieu sur la loi de l'aléa. Ce sont les statistiques qui, sur la base des informations recueillies, permettent de prévoir le destin moyen des personnes à assurer et de déterminer par voie de conséquence le niveau des primes à payer, en fonction de la « classe »de risques à laquelle elles appartiennent. La classification des risques est, pour l'assureur, un moyen de moduler ses tarifs et de proposer des primes différenciées aux «bons risques » et aux « mauvais risques », de façon à attirer les «bons risques » par des offres avantageuses dans un contexte de concurrence. Car l'assureur qui propose des primes calculées à partir d'un risque moyen va retenir les "mauvais risques », prêts à payer cette prime, mais faire fuir les «bons risques », qui la trouveront excessive. La concurrence pousse donc à la segmentation de la clientèle, et par voie de conséquence à la recherche, par les assureurs, du maximum d'informations permettant d'apprécier, avec la marge d'erreur la plus faible possible, le niveau de risque des candidats à l'assurance.

L'assurance repose aussi sur la mutualisation des risques, qui consiste à faire assumer par une collectivité le coût de la réalisation d'un risque individualisé peu fréquent mais d'impact important. Théoriquement, on peut imaginer une mutualisation globale de l'ensemble des risques, sur le modèle de la sécurité sociale, qui fonctionne selon un principe de solidarité, les «bons risques » subventionnant les «mauvais risques ». Mais lorsque la décision de s'assurer résulte d'une démarche individuelle et volontaire, chacun réclame de payer en fonction de son risque, les personnes les moins risquées cherchant, comme on l'a dit, des tarifs plus bas, de sorte que, dans un univers de marché, les assureurs sont amenés à tenir compte, pour fixer leurs tarifs, de l'appartenance de chaque individu à une classe d'équivalence en termes de risque (jeunes $v s$ vieux, malades $v s$ bien portants), la mutualisation des risques s'opérant au sein de cette classe d'équivalence.

Le troisième principe sur lequel se fonde l'assurance est la sélection des risques, principe qui est lui aussi directement lié à l'existence d'un marché concurrentiel et à la recherche du profit par les compagnies. La sélection des risques s'inscrit dans une logique de rentabilité financière qui implique de maximiser les recettes et de minimiser les dépenses prévisibles. Chaque assureur veut donc pouvoir accepter de couvrir un risque au prix où il l'entend, moyennant le cas échéant une forte surprime. Il veut surtout pouvoir refuser de couvrir un risque afin de lutter contre le phénomène dit de «l'anti-sélection ». On entend par là le comportement spontané mais rationnel qu'on prête aux candidats à l'assurance, qui seraient d'autant plus enclins à s'assurer contre un risque qu'ils pensent - ou qu'ils savent - que celui-ci a de fortes chances de se réaliser. Une entreprise qui ne sélectionnerait pas ses risques pourrait dans un premier temps gagner des parts de marché ; mais, compte tenu de «l'anti-sélection », elle attirerait nécessairement les " mauvais risques »; confrontée à de nombreux sinistres, elle serait contrainte de rehausser ses primes, chassant ainsi les « bons risques » de son portefeuille, jusqu'à être finalement évincée du marché.

\footnotetext{
: On trouvera un exposé particulièrement éclairant des principes économiques sur lesquels se fonde l'assurance de personnes et des conséquences qui en résultent dans le rapport du Conseil national du sida, "Assurance et VIHSIDA. Pour une assurabilité élargie des personnes et une confidentialité renforcée des données de santé », 20 septembre 1999, reproduit dans Ethique, sida et société, rapport d'activité du Conseil national du sida, Tome IV, 1999-2000, La Documentation française, 2001, pp. 19-116.
} 
La sélection des risques est donc présentée comme une nécessité économique, induite par la loi du marché.

\section{... qui renforce l'exclusion des plus vulnérables}

L'application de critères de sélection qui conduisent à écarter ceux qui risquent de coûter plus cher, de rapporter moins, ou de constituer un handicap face à la concurrence, équivaut en général à exclure les individus les plus vulnérables et à redoubler les phénomènes de stigmatisation à l'œuvre dans la société.

\section{Les formes d'exclusion classiques}

Dans les procédures de recrutement, on écartera, dans toute la mesure du possible, ceux que, à compétence égale, on soupçonne d'être moins productifs: les femmes, dont on craint les absences pour cause de grossesse ou de garde d'enfants, les personnes en moins bonne santé ou atteintes d'un handicap. On s'efforcera également d'anticiper les désirs, réels ou supposés, de la clientèle, en rejetant la candidature de ceux qui, par leur apparence physique notamment, seraient susceptibles de la faire se détourner : la couleur de la peau, l'accent, la boucle d'oreille, l'obésité, une infirmité quelconque, sont autant de stigmates qui vont dissuader de recruter des individus déjà victimes d'exclusion par ailleurs.

Ce type de tri tombe en grande partie, il est vrai, sous le coup de la loi pénale comme du code du travail, qui interdisent les discriminations fondées sur la race, l'apparence physique, le sexe, l'orientation sexuelle ou l'état de santé (v. infra, 3). Mais, quels que soient les progrès législatifs réalisés dans ce domaine, la preuve de la discrimination à l'embauche restera toujours difficile à apporter, car elle se dissout, soit dans la subjectivité du choix opéré par l'employeur, soit à l'inverse dans l'objectivité apparente d'une décision faisant intervenir une multiplicité de critères. À supposer même qu'elle parvienne à démontrer qu'il y a bien eu discrimination, au demeurant, la victime n'en tire pas un droit à être recrutée.

Les critères de sélection utilisés dans le champ de l'assurance ou du crédit diffèrent de ceux auxquels on a recours dans les procédures de recrutement. Les informations qui ont une incidence négative sur le «score » des emprunteurs n'en recouvrent pas moins assez largement les critères de la stigmatisation sociale : chômage, précarité, familles mono-parentales, endettement, etc. Quant à la sélection des risques dans le domaine de l'assurance-vie, essentiellement fondée sur l'état de santé, elle joue comme un facteur d'exclusion pour les personnes qui sont ou ont été atteintes d'affections considérées comme des «risques aggravés »: cancer, maladies cardiovasculaires, diabète. Lorsqu'elles demandent à souscrire une police d'assurance incluant la garantie des risques décès, invalidité ou arrêt de travail, elles risquent fort de se heurter à un refus ou, dans le meilleur des cas, de se voir proposer une couverture avec une forte surprime.

Cette discrimination dans l'accès à l'assurance est restée longtemps ignorée, ou du moins occultée, jusqu'à ce que le sida, servant ici, comme dans beaucoup d'autres domaines, de révélateur, fasse apparaître en pleine lumière les conséquences de la sélection des risques. Car les compagnies ont non seulement refusé d'assurer les personnes atteintes du sida, jugeant que, compte tenu de l'évolution fatale de la maladie, la condition d'aléa n'était pas remplie, mais elles ont également estimé que les personnes simplement séropositives n'étaient pas non plus assurables, dès lors qu'il y avait toutes les raisons de penser qu'elles développeraient la maladie à brève échéance. 
L'exclusion des séropositifs des mécanismes de droit commun, mal compensée, on le verra, par la mise en place d'un système d'assurance spécifique (v. infra, 3), illustre bien le cercle vicieux de la discrimination engendré par la recherche du profit: une fois admis que les personnes séropositives sont inassurables, il est logique que les compagnies cherchent à vérifier non seulement que le candidat à l'assurance n'est pas séropositif, mais encore qu'il n'appartient pas à un « groupe à risque », susceptible, par conséquent, d'être contaminé, ou même qu'il n'est pas en contact avec des personnes appartenant à un tel groupe. La concurrence, de son côté, pousse les compagnies à s'aligner progressivement sur celles qui pratiquent la sélection la plus rigoureuse pour ne pas supporter seules les risques les plus importants.

\section{La perspective d'une sélection génétique ${ }^{9}$}

Les progrès du diagnostic génétique, qui permet - ou permettra à terme - de prévoir la survenance d'une maladie avant l'apparition de tout symptôme chez une personne bien portante et sans antécédents familiaux, mais aussi de connaître les prédispositions génétiques d'un individu, d'évaluer le risque de survenue d'une affection déterminée, ouvre un champ nouveau à la sélection sur critères génétiques, notamment dans le domaine de l'assurance et de l'emploi.

La prise de conscience des risques de discrimination qui en découlent a incité le législateur français à encadrer le recours aux tests génétiques, dans les conditions qu'on rappellera plus loin. On voudrait néanmoins montrer ici comment la perspective d'une sélection génétique n'est pas pour autant définitivement écartée car, outre que certains pays l'ont autorisée, elle est en quelque sorte appelée par la loi du marché.

Pour l'employeur, la connaissance des caractéristiques génétiques des candidats à l'embauche permettrait de réduire l'incertitude sur l'avenir des différents candidats. Or on peut considérer que, lorsqu'une entreprise embauche et donc forme des salariés, elle investit, donc prend des risques, parmi lesquels figurent la maladie et la mort. Plus prosaïquement, la maladie du salarié et les absences qu'elle provoque, perturbe le fonctionnement de l'entreprise ${ }^{10}$. Dans cette perspective, la connaissance par l'employeur du risque futur d'altération de la santé du candidat le dissuadera de l'engager.

Dans l'état actuel de la législation, toutefois, il est clair que l'employeur ne peut solliciter du candidat à un emploi des informations concernant son état de santé, non plus que ses caractéristiques génétiques, car elles relèvent de sa vie privée. Reste que, sous couvert de prévention, il peut se produire qu'un salarié soit écarté d'un poste de travail pour lequel il présente une contre-indication médicale révélée par un diagnostic génétique". Le médecin du travail sera de plus en plus enclin à demander, parmi les examens permettant le diagnostic d'une

\footnotetext{
- Voir sur ce point : Dorothée Dibie, « Discriminations biologiques et droit des contrats » in C. Labrusse-Riou dir. Le droit saisi par la biologie. Des juristes au laboratoire, LGDJ, Bibl. de droit privé, 1996, p. 149 ; Marie-Isabelle Malauzat, Le droit face au pouvoir des données génétiques, Presses universitaires d'Aix-Marseille, 2000.

${ }^{10}$ La maladie n'est pas une cause légitime de licenciement, ce qui oblige à conserver son emploi au salarié qui ne peut être remplacé que temporairement. Le licenciement ne peut intervenir que si les absences répétées ou prolongées entraînent une perturbation telle au bon fonctionnement de l'entreprise que le remplacement définitif du salarié malade se révèle indispensable.

"V. Isabelle Vacarie, «Les discriminations dans l'emploi fondées sur les caractères génétiques », in CREDOFUniversité de Paris X, La lutte contre les discriminations, entre théorie et pratique, entre droit et politique, Actes de la journée d'études du 21 juin 2000.
} 
éventuelle contre-indication au poste de travail, l'examen des caractéristiques génétiques. Sur cette base, il sera le cas échéant amené à formuler un avis d'inaptitude, et cela, alors même que la maladie ne s'est pas déclarée, et, s'il s'agit d'un simple diagnostic de prédisposition, alors même qu'il n'indique qu'une probabilité et non une certitude que la maladie surviendra. Car si, par exemple, des salariés présentent, en raison de leurs caractéristiques génétiques, une plus grande susceptibilité à des agents chimiques cancérogènes, n'est-il pas du devoir du médecin du travail et, au-delà, de l'entreprise, de ne pas les y exposer?

Mais ces mesures de protection spécifiques, fondées sur la prise en compte des différences génétiques, ne risquent-elles pas de se substituer avantageusement, aux yeux de l'employeur, à des mesures de sécurité indistinctement applicables à tous les salariés et de déboucher sur une forme de sélection génétique des salariés ?' Il est moins coûteux, en effet, pour une entreprise, de ne pas recruter certains salariés pour occuper les emplois à risque que de mettre en place des politiques globales de prévention des risques et des maladies professionnelles d'améliorer les conditions d'hygiène et de sécurité.

Dans le champ de l'assurance, la tentation de connaître les caractéristiques génétiques du candidat à l'assurance sera à terme encore plus grande. Lorsque le risque couvert est la maladie ou le décès, toute information relative à l'évolution prévisible de l'état de santé du demandeur et à son espérance de vie est précieuse pour l'assureur. La prédiction génétique concourt à la détermination du risque, elle permet de mieux évaluer le risque à couvrir, et donc de fixer le montant de la prime de façon proportionnée au risque. Elle permet une classification plus fine des risques à garantir, poussant ainsi à une segmentation accrue du marché : les catégories d'assurés étant différenciées en fonction de leurs caractéristiques génétiques, les assureurs pourront baisser les primes des «bons risques» et augmenter les primes des «mauvais risques ». Elle permet enfin de lutter contre le phénomène d'anti-sélection, qui résulte de l'asymétrie de l'information entre le candidat et l'assureur ${ }^{13}$. Pour toutes ces raisons, on peut craindre que la prohibition posée par le législateur français (v. infra, 3) ne soit qu'un moratoire, qu'on n'hésitera pas à remettre en cause lorsque le diagnostic génétique, autorisé dans d'autres pays, sera devenu plus fiable et se sera banalisé.

La prédisposition génétique, qui est déjà en soi un fardeau lourd à porter, risque donc de constituer demain un véritable stigmate social, une nouvelle source d'inégalité face à l'assurance, mais aussi au crédit et à l'emploi. La sélection génétique fonctionnera bien comme un mécanisme de discrimination supplémentaire au détriment des plus vulnérables.

\footnotetext{
${ }^{12}$ D'où les vives réactions syndicales qu'a suscitées l'adoption, en 1997, par le conseil d'administration de 1'Institut National de Recherche et de Sécurité (INRS), d'un programme de travaux consacré à «la médecine de prévision appliquée au travail ». La problématique qui sous-tendait la recherche laissait en effet craindre qu'il ne débouche sur une sélection, pour des postes de travail particulièrement exposés, des seules personnes qui, en raison de leurs caractères génétiques, ne sont pas prédisposées à une maladie professionnelle, au détriment de mesures de sécurité applicables à l'ensemble des salariés.

"Marie-Isabelle Malauzat, op. cit. Voir aussi Marie-Angèle Hermitte, L’utilisation des tests génétiques par les assureurs, rapport ronéoté, GIP Justice, février 200066 p.
} 


\section{3/ Peut-on échapper à la « loi d’airain » du marché ?}

Si la « loi d'airain » du marché produit inéluctablement de la discrimination, toute intervention de l'État visant à en juguler les effets suppose tout aussi inéluctablement d'encadrer la liberté contractuelle et le jeu de la concurrence. Dire cela, c'est mettre le doigt sur les apories de la lutte contre les discriminations dans la sphère marchande : car jusqu'où peut-on restreindre la liberté contractuelle et la libre concurrence sans porter atteinte aux fondements mêmes de l'économie de marché ? Le législateur peut bien prohiber les discriminations les plus choquantes, réglementer les modalités de passation ou le contenu des contrats là où la dissymétrie entre les contractants est la plus flagrante, son intervention connaît forcément des limites. Une fois reconnue l'impossibilité d'éradiquer les discriminations dans le cadre du marché, il ne reste d'autre solution que de s'affranchir de la logique du marché, à chaque fois qu'elle entrave l'égal accès à des droits fondamentaux, pour faire prévaloir une logique de la solidarité.

\section{La prohibition des discriminations les plus choquantes}

Le législateur a progressivement renforcé l'arsenal répressif et en a étendu le champ d'application à des formes de discriminations toujours plus nombreuses. Mais ce mode d'intervention rencontre des limites. Les unes sont inhérentes à tout dispositif de répression, lourd à mettre en œuvre pour un bénéfice aléatoire. Les autres découlent de l'impossibilité de dénier aux entreprises le droit de sélectionner leurs clients ou leurs salariés sur des critères qui ne soient pas strictement impersonnels et objectifs, sauf à décider qu'elles sont tenues, à l'instar des services publics, à un strict principe d'égalité - ce qui reviendrait précisément à nier leur liberté contractuelle et la légitimité de choix opérés en fonction d'un impératif de profit ${ }^{4}$. Le statut ambigu des discriminations fondées sur l'état de santé fournit une bonne illustration de cette contradiction.

\section{Les discriminations interdites}

C'est la loi du 1" juillet 1972 contre le racisme qui a pour la première fois introduit dans le code pénal des dispositions réprimant la discrimination fondée sur l'origine ou l'appartenance à une ethnie, une nation, une race ou une religion déterminée. À ces critères sont venus s'ajouter successivement le sexe et la situation de famille en 1975, les mœurs en 1985, le handicap en 1989, l'état de santé en 1990. Le nouveau code pénal, entré en vigueur en 1994, y a inclus les discriminations fondées sur les opinions politiques ou les activités syndicales, la loi du 16 novembre 2001, relative à la lutte contre les discriminations, les discriminations fondées sur l'orientation sexuelle, l'apparence physique, le patronyme, l'âge, et la loi du 4 mars 2002, sur les droits des malades, les discriminations fondées sur les caractéristiques génétiques. Le code pénal définit la discrimination comme la distinction opérée entre des personnes physiques ou morales sur la base d'un des critères que l'on vient de rappeler. Elle ne tombe toutefois sous le coup de la loi pénale que si elle consiste en l'un des comportements énumérés à l'article 225-2, parmi lesquels figurent le refus de fournir un bien ou un service et les discriminations en matière d'emploi.

"Voir supra nos remarques sur « la liberté de discriminer ». 
Le code du travail a connu une évolution similaire. Depuis les lois Auroux de1982, qui y ont introduit pour la première fois des dispositions interdisant les discriminations, la liste des critères de discrimination et des comportements interdits a été progressivement allongée, sur le modèle du code pénal. La loi du 16 juillet 2001, transposant sur ce point les directives communautaires du 27 novembre 2000 (dite «directive emploi ») et du 29 juin 2000 (dite «directive race »), a également institué un renversement partiel de la charge de la preuve lorsque la victime fait état d'éléments laissant présumer le caractère discriminatoire de la décision de l'employeur.

La loi de modernisation sociale du 17 janvier 2002, enfin, a introduit dans la loi de 1989 sur les rapports locatifs une disposition expresse qui interdit de refuser la location d'un logement pour l'un des motifs énumérés par le code pénal, et qui prévoit, comme en matière d'emploi, un renversement partiel de la charge de la preuve.

L'ensemble de ce dispositif n'a cependant qu'un impact très marginal sur le phénomène qu'il entend conjurer. La lourdeur de l'action en justice, le caractère aléatoire du bénéfice escompté, les difficultés de preuve, enfin, qui subsistent en dépit des progrès législatifs et jurisprudentiels

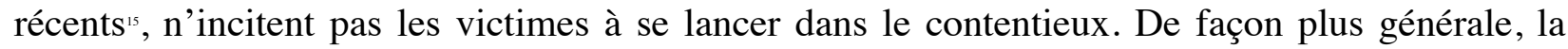
discrimination prohibée passe facilement inaperçue dans un environnement qui, comme on l'a rappelé plus haut, non seulement autorise la discrimination mais fonctionne à base de discrimination.

\section{Le statut ambigu des discriminations fondées sur l'état de santé}

C'est pour «mieux protéger les droits des malades et des handicapés » et « mieux prévenir les phénomènes d'exclusion ou de discrimination» que les pouvoirs publics, alertés sur les difficultés rencontrées dans leur vie quotidienne par les personnes touchées par le VIH, ont décidé, par la loi du 12 juillet 1990, de sanctionner pénalement les discriminations fondées sur l'état de santé, en particulier dans le cadre de la fourniture de biens et de services ou dans le cadre de l'emploi.

Mais les exceptions apportées par le législateur à l'interdiction des discriminations fondées sur l'état de santé réduisent à peu de chose la protection accordée. La première, aisée à justifier et difficilement évitable, concerne les refus d'embauche et les licenciements lorsqu'ils résultent du constat, par le médecin du travail, de l'inaptitude médicale du salarié. La seconde exclut l'assurance de personnes du champ d'application de la loi pénale'

Il faut savoir que cette exclusion, qui ne figurait pas dans le projet initial, a été introduite à la demande expresse des compagnies d'assurance. Pourtant, même sous sa forme initiale, le texte n'excluait pas toute sélection des risques, puisqu'il prévoyait la possibilité d'invoquer un motif légitime pour justifier le refus de fournir un bien ou un service : il aurait simplement obligé les compagnies à préciser les motifs du refus, introduisant un peu plus de transparence dans un domaine où l'opacité reste la règle. En reconnaissant aux assureurs, plus explicitement qu'aucun

\footnotetext{
"Sn pense notamment à l'admission par la Cour de cassation de la licéité de la preuve par «testing » en matière pénale.

"Dans sa rédaction actuelle, le nouveau code pénal prévoit que les discriminations fondées sur l'état de santé ne sont pas punissables «lorsqu'elles consistent en des opérations ayant pour objet la prévention et la couverture du risque décès, des risques portant atteinte à l'intégrité physique de la personne ou des risques d'incapacité de travail ou d'invalidité » (art. 225-3).
} 
texte ne l'avait jamais fait, le droit de sélectionner les candidats à l'assurance ou de fixer le montant des primes sur des critères de santé, en cédant à la pression d'un groupe économiquement puissant, le gouvernement a fait la démonstration que la lutte contre les discriminations pouvait être mise en échec par la loi d'airain du marché. Il a également réussi ce paradoxe qui consiste à entériner l'exclusion des personnes malades de l'accès à l'assurance dans une loi qui prétendait les protéger contre les discriminations !

En ce qui concerne l'interdiction de la prise en compte des caractéristiques génétiques, en revanche, l'opposition des assureurs n'a pas été aussi déterminée. Il est vrai que le diagnostic génétique n'est encore ni suffisamment généralisé, ni suffisamment performant pour être utilisé par les assureurs comme outil de sélection des risques dans leur pratique courante. Dès le début de 1994, de fait, la Fédération française des sociétés d'assurances a d'elle-même adopté un moratoire de cinq ans, reconduit en mars 1999, sur les examens génétiques.

Avant que la loi du 2 mars 2002 sur les droits des malades ne vienne trancher la question, les avis concernant, tant la licéité de la sélection sur critères génétiques au regard des textes en vigueur que l'opportunité de les interdire, restaient très partagés. Sans doute les lois bioéthiques de 1994 ont-elles exclu la réalisation de tests génétiques pour une finalité autre que médicale ou de recherche scientifique, et sanctionné pénalement le détournement des informations ainsi recueillies ; mais il n'était pas évident que soit assimilable à un détournement la communication à l'assureur, par le candidat à l'assurance, du résultat d'un test réalisé pour des raisons médicales. Sans doute aussi la Convention du Conseil de l'Europe sur les droits de l'homme et la biomédecine (Convention d'Oviedo, 1997) interdit-elle «toute forme de discrimination à l'encontre d'une personne en raison de son patrimoine génétique » (art. 11), interdiction qu'on retrouve dans la Déclaration universelle sur le génome humain et les droits de l'homme de 1997, qui proclame que «nul ne doit faire l'objet de discriminations fondées sur ses caractéristiques génétiques, qui auraient pour objet ou pour effet de porter atteinte à ses droits individuels et à ses libertés fondamentales et à la reconnaissance de sa dignité » (art. 6). Mais on pouvait objecter que la sélection des risques n'est pas constitutive d'une discrimination, dès lors qu'elle est au fondement de l'assurance.

Le Comité consultatif national d'éthique s'était clairement prononcé, en 1995, pour une interdiction totale de l'utilisation des données génétiques à des fins de sélection ou de discrimination, que ce soit dans le domaine des politiques de santé, de l'emploi ou des systèmes d'assurance". Mais le Conseil d'État, pour sa part, faisait valoir que l'assureur étant déjà en droit de solliciter des renseignements médicaux pour évaluer les risques qu'il prend en charge, la prise en compte des tests génétiques ne constituerait qu'une simple différence de degré et non de nature. Écartant l'idée d'une interdiction absolue, il optait pour une solution intermédiaire, consistant à interdire aux compagnies d'assurance d'imposer à leurs souscripteurs de se soumettre à des tests, mais admettant qu'un souscripteur pourrait être tenu de produire le résultat d'un test de prédisposition qu'il aurait subi volontairement avant la conclusion de son contrat $^{18}$.

\footnotetext{
${ }^{17}$ Avis du 30 octobre 1995 :« Génétique et médecine : de la prédiction à la prévention ».

${ }^{18}$ Conseil d'État, Les lois de bioéthique : cinq ans après, 1999. p. 118 et s.
} 
La loi du 4 mars 2002, qui complète le code du travail et le code pénal en prohibant toute discrimination fondée sur les caractéristiques génétiques, interdit clairement le recours aux tests génétiques dans le champ de l'assurance, généralisant ainsi l'interdiction déjà posée par la loi CMU de 1999 pour les organismes de protection complémentaire. Désormais, l'article 1141-1 du code de la santé publique, auquel renvoie l'article L. 133-1 du code des assurances, interdit aux assureurs qui proposent une garantie des risques d'invalidité ou de décès de tenir compte des résultats de l'examen des caractéristiques génétiques, même si ceux-ci leur sont transmis par la personne concernée ou avec son accord. Il leur est également interdit de poser aucune question relative aux tests génétiques et à leurs résultats ou de demander à une personne de se soumettre à des tests génétiques, que ce soit avant la conclusion du contrat ou pendant la durée de celui-ci. La violation de ces interdictions est pénalement réprimée : la loi ajoute à l'article 225-3 du code pénal, qui autorise les assureurs à opérer des discriminations fondées sur l'état de santé, une disposition prévoyant que la discrimination est en revanche constitutive d'un délit lorsqu'elle se fonde «sur la prise en compte de tests génétiques prédictifs ayant pour objet une maladie qui n’est pas encore déclarée ou une prédisposition génétique à une maladie ».

Reste à savoir si cette interdiction résistera longtemps à la pression des intérêts économiques et à la logique de la sélection des risques, alors que les compagnies d'assurance sont en concurrence au niveau européen et que certains pays, comme la Grande-Bretagne, ont accepté la prise en compte des tests génétiques ${ }^{19}$.

\section{La solidarité contre le marché}

Si la logique du marché non seulement est incapable de produire de l'égalité, mais engendre de nouvelles formes de discrimination que l'encadrement de la liberté contractuelle ne suffit pas à juguler, force est de recourir à d'autres modes de régulation si l'on ne veut pas laisser la réalisation des droits fondamentaux dépendre des aléas du marché.

Parmi les droits les plus directement menacés par la loi du marché figurent, on l'a vu, le droit à l'emploi et le droit à la santé, garantis par le Préambule de 1946, le droit au logement, proclamé à plusieurs reprises par le législateur, mais aussi - il convient de s'y arrêter un instant - la possibilité de s'assurer. Car s'il n'existe pas à proprement parler de droit à l'assurance, l'assurance est devenue, directement ou indirectement, la condition de l'exercice d'une série de droits fondamentaux, de sorte que l'impossibilité d'accéder à l'assurance équivaut à être privé de ces droits. Le droit au logement, l'exercice d'une activité professionnelle et donc la liberté d'entreprendre, le droit de disposer des biens de consommation indispensables, sont de plus en plus souvent conditionnés par le recours à l'emprunt; or l'accès au crédit est lui-même conditionné par l'accès à l'assurance, puisque les banques exigent que le remboursement du prêt soit garanti par une assurance couvrant les risques décès, invalidité et maladie.

L'émotion suscitée, en février 2000, par la décision de la compagnie Axa de doubler les cotisations des contrats souscrits par les parents d'enfants handicapés pour garantir, après leur décès, une rente à leurs enfants, fournit une illustration supplémentaire du rôle vital joué par certaines formes d'assurance. Mais cette affaire a aussi constitué l'aveu, de la part d'un

19 Voir sur ce point l'étude détaillée réalisée par Christine Noiville et inclue dans le rapport de Marie-Angèle Hermitte précité. 
organisme géré à l'aune du profit, de son incapacité à garantir à tous, et pas seulement aux plus fortunés, l'accès aux prestations qu'il offre ${ }^{20}$.

Avec le désengagement programmé de l'État, ce type de problèmes risque de se poser avec une acuité croissante. L'existence, en France, d'un système de sécurité sociale obligatoire et universel a permis jusqu'à présent de faire échapper la protection sociale à la loi du marché et à la sélection des risques. Mais la part des assureurs privés dans le secteur de la protection sociale complémentaire est appelée à s'accroître, et les compagnies d'assurances revendiquent désormais d'être présentes dans le financement et la gestion de l'assurance maladie, à côté, voire à la place des systèmes publics ou mutualistes ${ }^{21}$ : la logique du marché ne risque-t-elle pas de contaminer la logique de la solidarité ? La crainte n'est pas vaine, car l'expérience des pays étrangers qui ont introduit la concurrence dans le secteur de l'assurance maladie, comme les États-Unis et les PaysBas, montre qu'en dépit d'une réglementation stricte visant à éviter la sélection des risques (obligation d'accepter toute demande d'adhésion, compensation des risques entre les caisses), l'organisation concurrentielle pousse malgré tout inéluctablement à des formes de sélection indirecte, aboutissant soit à l'exclusion du système d'une partie de la population, soit à des inégalités dans les prestations et la qualité de service offertes en fonction de l'état de santé ${ }^{22}$.

Si la question de l'assurance mérite à ce point qu'on s'y attarde, c'est bien sûr parce que les discriminations s'expriment et se déploient dans le secteur de l'assurance privée plus ouvertement et plus librement que nulle part ailleurs. C'est également parce que, compte tenu des besoins auxquels elle répond, de la fonction économique et sociale désormais essentielle qu'elle remplit, les discriminations dans l'accès à l'assurance emportent avec elles d'autres discriminations.

Mais l'assurance est aussi une technologie ${ }^{23}$, un mécanisme susceptible d'applications différenciées, dont il faut s'attacher à saisir l'ambivalence. Intégrée à une logique de marché, elle semble décidément incapable de produire autre chose que de la discrimination et de l'exclusion ; mais elle peut fonctionner, à l'inverse, « comme une sorte de main invisible de la solidarité $»^{24}$. L'État providence s'est ainsi développé sur la base d'un système assuranciel couvrant les principaux « risques » de l'existence : maladie, chômage, retraite, invalidité, et instaurant une solidarité entre tous les membres de la société, malades et bien-portants, pauvres et riches, inactifs et actifs.

Techniquement, pour que l'assurance produise de la solidarité, il faut accepter une forme de subvention croisée entre bons risques et mauvais risques, et donc faire prévaloir trois principes, que seul l'État est capable d'imposer : une tarification uniforme, l'interdiction pour les assureurs de refuser de couvrir les «mauvais risques», et l'obligation pour les «bons risques» de s'assurer, de manière à garantir l'assiette de la redistribution ${ }^{25}$. Autrement dit, aller à contre-

${ }^{20}$ Le Monde, 18 février 2000, «L'aveu d'Axa ».

${ }^{21}$ On trouvera une présentation de ces projets qui visent à réformer, et même à transformer radicalement l'assurance santé dans la revue Risques. Les cahiers de l'assurance, $\mathrm{n}^{\circ} 49$, janvier-mars 2002.

${ }_{22}$ Voir G. Demange, P.-Y. Geoffard, «Quelles formes de concurrence en assurance santé ? », dans le numéro précité de la revue Risques.

${ }^{23}$ François Ewald, L'État providence, Grasset, 1986, p. 171.

${ }_{24}$ Pierre Rosanvallon, La nouvelle question sociale. Repenser l’État providence, Seuil, 1995, p. 19.

${ }^{25}$ Christian Gollier, « Assurance et solidarité », Le Monde, 29 février 2000. 
courant de la tendance à l'hyper-segmentation des tarifs, fondée sur une appréciation fine de la probabilité du risque de chacun.

Le choix qui consisterait à « jouer la carte de la solidarité entre bonnes et mauvaises classes de risques, donc jouer la carte de l'opacité sans se renseigner sur le profil des souscripteurs $»^{26}$, a-t-il des chances de l'emporter ? On peut en douter. Ainsi, c'est la philosophie inverse qui inspire le dispositif récemment mis en place pour couvrir les risques de santé aggravés, dans le sillage de la convention sur l'assurabilité des personnes séropositives, signée en septembre 1991 entre les assureurs et l'État. Les concessions consenties par les pouvoirs publics aux compagnies auxquelles était reconnu le droit à la fois de sélectionner les candidats en fonction de leur état de santé et d'exiger d'eux un test de séropositivité - aboutissant à exclure les séropositifs des dispositif de droit commun, la convention leur ouvrait la possibilité, moyennant supprime, de souscrire un contrat d'assurance en cas de décès pour garantir un prêt immobilier. Cette convention a été un échec, pour une série de raisons dont les plus évidentes sont la limitation des garanties offertes (n'étaient couverts ni les prêts à la consommation, ni le risque invalidité), le montant trop élevé de la surprime, mais aussi la réticence aisément compréhensible à recourir à un dispositif par définition stigmatisant.

C'est pourtant ce modèle qui a été repris et développé par la convention du 19 septembre 2001 sur l'assurance des personnes présentant des risques de santé aggravés. Transversale à l'ensemble des pathologies, elle inclut cette fois les prêts à la consommation. Elle permet aussi de proposer des prêts immobiliers avec surprime aux personnes considérées comme inassurables, grâce à la mutualisation des risques entre les assureurs par la mise en place d'un "pool de risques aggravés » et d'un « pool de risques très aggravés ».

Faut-il voir dans ce dispositif, entériné par la loi du 4 mars 2002 relative aux droits des malades $^{27}$, une incursion de la solidarité dans le monde de l'assurance, comme on l'a dit, et la preuve qu'on peut concilier marché et solidarité ? Ne signe-t-il pas au contraire l'échec de la solidarité ? Consacrant la mise à l'écart des personnes malades ou qui ont été victimes d'une affection grave des dispositifs de droit commun, il repose en effet sur une double discrimination : entre malades et bien portants, puisque les conditions d'assurance offertes aux personnes couvertes par la convention sont beaucoup moins avantageuses que celles dont bénéficie le reste de la population ; entre riches et pauvres, puisque seuls les plus fortunés pourront s'assurer aux tarifs proposés, tandis que les autres, dans l'incapacité de payer les surprimes demandées, resteront exclus de l'accès à l'assurance.

La solution adoptée aujourd'hui pour les risques aggravés pourrait bien servir demain de modèle pour la couverture d'autres «mauvais risques », ouvrant ainsi la voie à une segmentation toujours plus forte du marché de l'assurance. L'hypothèse est d'autant plus plausible que la pression des intérêts économiques n'est pas la seule à pousser en ce sens. Le progrès de la

\footnotetext{
${ }^{26}$ Marie-Angèle Hermitte, op. cit.

${ }^{27}$ L'article L. 1141-2 du code de la santé publique auquel renvoie l'article L. 133-1 du code des assurances prévoit : «Une convention relative à l'assurance des personnes exposées à un risque aggravé du fait de leur état de santé détermine les modalités particulières d'accès à l'assurance contre les risques d'invalidité ou de décès en faveur de ces personnes qui ne peuvent trouver dans le cadre des pratiques habituelles de l'assurance de garantie des prêts à la consommation, immobiliers ou à caractère professionnel ».
} 
connaissance des inégalités naturelles entre les hommes, la « déchirure du voile d'ignorance ${ }^{28}$, sous l'effet des progrès de la médecine, notamment de l'épidémiologie et du diagnostic génétique, pourrait bien miner à la base le principe d'une mutualisation universelle qui présupposait que les individus étaient égaux devant les différents risques sociaux susceptibles d'affecter l'existence, et, à terme, faire voler en éclats l'ensemble de notre système de protection sociale $^{29}$.

Si cette perspective pessimiste se confirmait, elle signifierait la double victoire du marché : victoire matérielle, le consacrant comme mode hégémonique de régulation sociale ; mais aussi victoire intellectuelle et morale, puisqu'il apparaîtrait alors en pleine lumière que la loi du marché a inscrit profondément sa marque en chaque individu, et que cinquante ans d'État providence n'ont pas réussi à déraciner la propension à raisonner, bien au-delà de la sphère marchande, en termes de coût-bénéfice.

\footnotetext{
${ }^{28}$ Pierre Rosanvallon, op. cit., p. 54 et s.

" « "Face aux nouvelles inégalités révélées par la médecine prédictive, il faudra à nos sociétés du XXI siècle beaucoup d'héroïsme et beaucoup d'imagination pour réinventer une nouvelle solidarité », écrivent François Bourguignon et Jean-Jacques Duby, « Médecine prédictive. Nouvelles inégalités ou nouvelle solidarité ? », Risques, $n^{\circ} 21$, janvier-mars 1995, p. 125.
} 